\title{
As Equações dos Estados Quase-Permanentes e uma Visão Alternativa da Indução Eletromagnética
}

The equations of the quasi-steady states and an alternative view of the electromagnetic induction

\author{
G. F. Leal Ferreira \\ guilherm@if.sc.usp.br \\ Instituto de Física de São Carlos, USP \\ Caixa Postal 369, 13.560-970, São Carlos, S.P.
}

Recebido em 21 de Novembro 2000. Manuscrito revisado recebido em 25 de Julho 2001.

Aceito em 16 de Outubro de 2001.

\begin{abstract}
Apresenta-se o sistema de equações que descreve consistentemente os estados quase-permanentes, campos e potenciais, abrangendo a eletrostática, a magnetostática e a lei de indução de Faraday. Mostra-se que ele é correto até a ordem de $v^{2} / c^{2}$ e de $w r / c^{2}$ em que $v$ e $w$ são a velocidade e a aceleração tópicas das cargas, $c$ a velocidade da luz e $r$ a distância ao ponto considerado. Podese então obter o campo elétrico de uma carga em movimento nessa aproximação, o qual corrige o campo de Coulomb por termos dependentes da velocidade e da aceleração da carga. Mostrase assim, como feito anteriormente por Ritz e O'Rahilly, que a indução eletromagnética pode ser atribuída à aceleração das cargas do circuito indutor, propiciando uma visão alternativa dessa interação, usualmente atribuída à variação do fluxo no interior do circuito.
\end{abstract}

The equations of the quasi-steady states, for the fields and the potentials, covering electrostatics, magnetostatics and the Faraday Induction Law, are given. They are correct to the order of $v^{2} / c^{2}$ and $w r / c, v$ and $w$ being the typical velocity and acceleration of the charges, $c$ the speed of light and $r$ the distance to a given point . This last fact allow us to obtain, in this approximation, the electric field of a moving charge, which corrects the Coulomb field by terms depending on the velocity and acceleration of the charge. Then, it may be shown, as done previously by Ritz and O Rahilly, that electromagnetic induction may be attributed to the acceleration of the charges of the inducting circuit, thus ensuing an alternative view of this interaction, usually attributed to flux variation enclosed by the circuit.

\section{Introdução}

O ensino do eletromagnetismo começa com a eletrostática, vai à magnetostática, incorpora depois a lei de indução de Faraday, com a qual estuda a interação entre circuitos elétricos, sob o título de estados quase-permanentes (ou quase-estacionários) e finalmente chega às equações de Maxwell [1-4]. Atingido este último estágio, não se preocupa porém em estabelecer claramente a aproximação envolvida no estágio anterior, isto é, a dos estados quase-permanentes, ao qual dedicou tempo considerável, já que com ela muitas questões práticas foram estudadas (carga do condensador, o capítulo de correntes alternadas, motores etc). Vejamos o que diz o muito popular Reitz, Milford, e Christy [2]. No Cap. 13, estudando correntes variando lentamente no tempo, diz, em nota de roda-pé, que os estados quase- permanentes resultam da aproximação de se desprezar no rotacional do campo de indução magnética a contribuição da corrente de deslocamento. Como notou há muito Maxwell, isto tornaria aquela equação inconsistente. Do nosso conhecimento, só em [5] o assunto é discutido explicitamente, mas de forma inadequada (ver seção III adiante). Parece-nos, portanto, interessante e mesmo necessário esclarecer o assunto, o que, aliás, é muito simples de ser feito, como veremos na seção II, em que o sistema de equações dos campos elétrico e de indução magnética é apresentado. Na seção III os correspondentes potenciais são dados e na seção IV estuda-se em que região e em que condições a aproximação fornece valores quase corretos. É, na verdade, aqui que o presente estudo se mostra especialmente frutífero: ele permitirá obter, nessa aproximação, o campo coulombiano corrigido pela velocidade e pela aceleração da carga em movimento, estudado na seção V. Poderemos, então, penetrar na ainda penumbrosa região do ensino atual do Eletromagnetismo, aquele da indução eletromagnética, no 
qual se procura atribuir seus efeitos a uma ação coletiva dos circuitos - variações do fluxo magnético de um circuito no interior de outro circuito, seguindo a tradição Faraday-Maxwell -, sem nenhuma referência às fontes, as cargas elétricas em movimento, em insuspeitado contraste com a metodologia empregada no ensino da eletrostática e da magnetostática. Como mostraram anteriormente Ritz [6] e O'Rahilly [7], que derivaram seus resultados dos potenciais de LiénardWiechert, a indução eletromagnética deve ser atribuida à aceleração das cargas do circuito indutor, reconciliando sua metodologia com a do ensino da eletrostática e da magnetostática. Trata-se de uma reunificação conceitual importante, impossível de ser feita sem a visão eletrônica -inexistente no tempo de Maxwell-, mas que não pode ser ignorada hoje em dia. E que por ser praticamente desconhecida da literatura, reproduzimos aqui, na seção VI.

\section{O sistema de equações dos es- tados quase-permanentes}

Como dissemos há pouco, a afirmativa de Reitz, Milford e Christy de que os estados quase-permanentes resultam da aproximação de se desprezar a corrente de deslocamento na equação do rotacional do campo de indução não está correta porque gera uma equação inconsistente. Como notou Maxwell, tomando-se a divergência de ambos os lados da equação, a divergência do rotacional é identicamente nula enquanto que a divergência da densidade da corrente não o é. Na verdade, a aproximação dos estados quase-permanentes (EQ-P) consiste em usar-se, naquela equação, não o vetor deslocamento, mas o campo eletrostático. Aliás, é assim que se inicia o ensino da indução eletromagnética: ao campo elestrostático $\vec{h}$ superpomos o campo elétrico solenoidal $\vec{S}$. Teríamos, então, as seguintes equações, incorporando o conteúdo da lei de Faraday, a eletrostática e a magnetostática

$$
\begin{gathered}
\nabla \cdot \vec{h}=4 \pi \rho, \\
\nabla \times \vec{h}=0,
\end{gathered}
$$

sendo $\rho$ a densidade de cargas, e para o campo solenoidal $\vec{S}$ com

$$
V \times \vec{S}=-\frac{1}{c} \frac{\partial \vec{B}}{\partial t}
$$

e, naturalmente,

$$
\nabla \cdot \vec{S}=0
$$

sendo $c$ a velocidade da luz, e $\vec{B}$ o campo de indução magnética, e o campo elétrico total, $\vec{E}$, é

$$
\vec{E}=\vec{h}+\vec{S}
$$

A dependência dos campos na posição $\vec{x}$ e no tempo $t$ está sendo omitida na notação e estamos usando o Sistema CGS Gaussiano. Para o campo $\vec{B}$ definimos

$$
\begin{gathered}
\nabla \cdot \vec{B}=0 \\
\nabla \times \vec{B}=\frac{4 \pi}{c}\left(\vec{j}_{c}+\frac{1}{4 \pi} \frac{\partial \vec{h}}{\partial t}\right)
\end{gathered}
$$

ligando $\vec{B}$ às densidades de corrente de condução, $\vec{j}_{c}$, e fazendo a Eq.7 auto-consistente, tendo em vista a validade da equação da continuidade

$$
\nabla \cdot \vec{j}_{c}+\frac{\partial \rho}{\partial t}=0
$$

As Eqs. 1-8 constituem o sistema de equações dos estados quase-permanentes, cobrindo o Eletromagnetismo em que os campos estão situados nas proximidades das cargas e correntes, como logo veremos. É interessante notar que ele difere do sistema de equações de Maxwell exclusivamente pela presença na Eq.7 de $\vec{h}$ em vez de $\vec{E}$, campo elétrico total, Eq.5. No caso das Equações de Maxwell exatas, a separação de $\vec{E}$ em $\vec{h}$ e $\vec{S}$ deixa de ser necessária. Uma hipótese adicional deve ser feita para se estudar o campo de cargas em movimento, como faremos adiante. É ela

$$
\vec{j}_{c}=\rho \vec{v}
$$

sendo $\vec{v}$ a velocidade das cargas.

\section{Os Potenciais dos EQ-P}

Mostraremos que os potenciais escalar, $U$, e vetorial, $\vec{A}$, definidos pelas relações

$$
U(\vec{x}, t)=\int \frac{\rho\left(\vec{x}^{\prime}, t\right) d V^{\prime}}{\left|\vec{x}-\vec{x}^{\prime}\right|}
$$

e

$$
\vec{A}(\vec{x}, t)=\frac{1}{c} \int \frac{\left(\vec{j}_{c}\left(\vec{x}^{\prime}, t\right)+\frac{1}{4 \pi} \frac{\partial \vec{h}\left(\vec{x}^{\prime}, t\right)}{\partial t} d V^{\prime}\right.}{\left|\vec{x}-\vec{x}^{\prime}\right|}
$$

sendo $d V^{\prime}$ o diferencial de elemento de volume, geram os campos dos E-QP dados na seção anterior, Eqs.1-8, através das prescrições usuais

$$
\vec{E}=-\nabla U-\frac{1}{c} \frac{\partial \vec{A}}{\partial t}
$$

e

$$
\vec{B}=\nabla \times \vec{A}
$$

Notemos primeiro que, pelas Eqs.11 e 8, temos

$$
\nabla \cdot \vec{A}=0
$$

e, pelas Eqs.12, 10 e 14, acha-se

$$
\nabla \cdot \vec{E}=\nabla \cdot \vec{h}=4 \pi \rho
$$


Agora, através das Eqs.12, 13 e 5, obtém-se

$$
\nabla \times \vec{B}=\nabla \times \vec{S}=-\frac{1}{c} \frac{\partial \vec{B}}{\partial t}
$$

sendo

$$
\vec{S}=-\frac{\partial}{c} \frac{\partial \vec{A}}{\partial t}
$$

Para o campo magnético temos, pela Eq.13

$$
\nabla \cdot \vec{B}=0
$$

e para o rotacional, através da identidade

$$
\nabla \times \nabla \times \vec{A}=-\nabla^{2} \vec{A}+\nabla \nabla \cdot \vec{A}
$$

obtém-se, com o auxílio da Eq.14,

$$
\nabla X \vec{B}=\frac{4 \pi}{c}\left(\vec{j}_{c}+\frac{1}{4 \pi} \frac{\partial \vec{h}}{\partial t}\right)
$$

equações que reproduzem as Eqs.1, 36 e 7, tendo em conta a Eq.5. Pode ser facilmente verificado que se na Eq.11, definindo o potencial vetor, tivéssemos adicionado ao campo eletrostático $\vec{h}$ o campo solenoidal $\vec{S}$, obteríamos as Equações de Maxwell exatas. Note-se que, em princípio, poderíamos realizar transformações de calibre [1-3] nos potenciais $U$ e $\vec{A}$, dados nas Eqs.10 e 11 , mantendo os valores de $\vec{E}$ e $\vec{B}$ inalterados. Mas a separação do campo elétrico numa parte eletrostática, Eqs.1 e 2, e outra solenoidal, Eqs.3 e 4, de grande apelo físico, ficaria comprometida. Pode-se mostrar que se partirmos de ambos potenciais como instantâneos (e não só o escalar como na Eq.10),- que é como os EQ$\mathrm{P}$ são definidos em [5] -, isto não corresponde a uma transformação de calibre realizada sobre os $U$ e $\vec{A}$ das Eqs. 10 e 11, e, assim, não podem ser considerados como ponto de partida alternativo para os EQ-P.

\section{Ordem de Aproximação das EQ-P}

Uma questão importante é a da aproximação incubada nas equações dos EQ-P. Para respondê- la, devemos estudar com que aproximação se obtêm os campos de uma carga $q$, com velocidade $v$ e aceleração $w$. Viu-se que a aproximação dos EQ-P consiste em se usar $\vec{h}$ em vez de $\vec{E}$ na Eq.11, desprezando-se $\vec{S}$, Eq.5. Como $S$ é $1 / c$ vezes a derivada temporal de $A$, Eq.17, (tratando-se de estimativas só os módulos das grandezas serão referidos) e $A$ é da ordem de $q v / c r, S$ será da ordem de $q v^{2} / c^{2} r^{2}$ e de $q w / c^{2} r$, que, aliás, será também a ordem com que o campo elétrico será calculado, Eq.12, onde $r$ é a distância da carga ao ponto considerado. Mas a inclusão de $S$ junto a $h$, que é da ordem de $q / r^{2}$, no integrando da Eq.11 geraria termos de ordem superior àqueles com que $E$ é calculado e portanto concluímos que as equações dos EQ-P fornecem o campo elétrico correto até termos da ordem de $q v^{2} / c^{2} r^{2}$ e $q w / c^{2} r$. Dentro do espírito de uma aproximação, as correções serão suficientes se forem muito menores do que o termo dominante, que é o campo coulombiano. Vemos, então, que $r<<c^{2} / w$. Com relação ao campo magnético, como correntes eletrostáticas de deslocamento $(\partial \vec{h} / \partial t)$ não criam campo magnético ([8] ou ver Apêndice), ele, da Eq.13, será da ordem de $q v / c r^{2}$, mas que dá no cálculo de forças (de Lorentz), termo também da ordem de $q v^{2} / c^{2} r^{2}$, isto é, compatível com aquela com que é dado o campo elétrico.

Aproximações para os campos de uma carga em movimento podem ser obtidos alternativamente a partir dos potenciais de Liénard-Wiechert [1-3], como realizado por Ritz [6] e O'Rahilly [7], e daí ser interessante comparar resultados obtidos de pontos de partida aparentemente tão distantes. O cálculo segundo as equações dos EQ-P está realizado no Apêndice e seu resultado concorda com aquele fornecido por Ritz e O'Rahilly, via Liénard- Wiechert. Obtêm-se

$$
\vec{E}=\frac{q}{r^{2}}\left[\hat{r}\left(1 \frac{\vec{v}^{2}-3(\vec{v} \cdot \hat{r})^{2}}{2 c^{2}}-\frac{\vec{w} \cdot \vec{r}}{2 c^{2}}\right)-\frac{r \vec{w}}{2 c^{2}}\right]
$$

$$
\vec{B}=\frac{q \vec{v} \times \vec{r}}{c r^{3}}
$$

em que $\vec{r}$ é o vetor posição do ponto do campo, contado da posição instantânea da carga $q$, com velocidade $\vec{v} \mathrm{e}$ aceleração $\vec{w}$. $\hat{r}$ é o versor de $\vec{r}$.

\section{Breve estudo do campo elétrico dos EQ-P para a carga pontual}

Daqui em diante, chamaremos de campo elétrico dos EQ-P a parte em excesso ao campo puramente coulombiano, ou seja, o campo dependendo somente da velocidade, $\vec{E}_{\vec{v}}$, e o campo $\vec{E}_{\vec{w}}$, dependendo somente da aceleração $\vec{w}$ da carga em movimento. Na Fig.1, mostramos a dependência angular de $r^{2} \vec{E}_{\vec{v}}$, como funcão de $x$ e $y$, para o caso de velocidade na direção $+x$. É um campo radial, de caráter rotacional bem visível (ele obedece à Eq.16), e vê-se que ele diminui o campo na direção do movimento e o aumenta na direção perpendicular. Enquanto que o campo de velocidade $\vec{E}_{\vec{v}}$ cai com $1 / r^{2}$, o campo de aceleração $\vec{E}_{\vec{w}}$ cai como $1 / r$. A dependência angular de $r . \vec{E}_{\vec{w}}$ está mostrada na Fig.2 para o caso de aceleração na direção $+x$. 


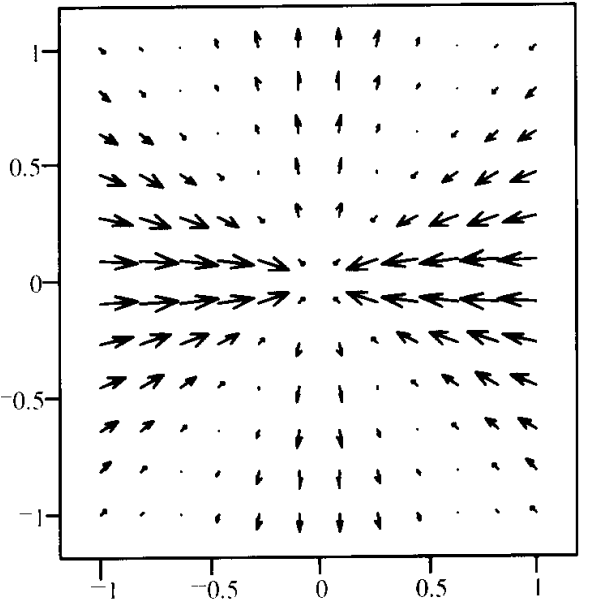

$X, Y$

Figura 1. Padrão de linhas de campo de $r^{2} \vec{E}_{v}$, Eq.21, calculado com as facilidades do MathCad plus 6. A carga com velocidade constante está em $x, y=0$ movendo-se na direção $+x$.

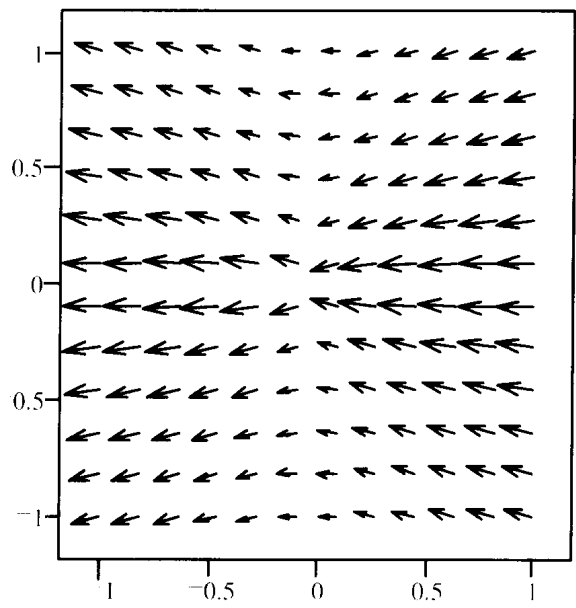

$X, Y$

Figura 2. Padrão de linhas de campo de $r \cdot \vec{E}_{w}$, Eq.21, calculado com as facilidades do MathCad plus 6. A carga em $x, y=0$, tem aceleração para a direita.

\section{A indução eletromagnética}

Como comentado na Introdução, na apresentação usual [1-4] a indução eletromagnética é atribuida à variação no tempo do fluxo do campo $\vec{B}$ 'aprisionado' num circuito fechado (às vezes, apenas se imagina que é fechado e calcula-se a variação de um fluxo imaginário [9], ver seção 7). Alternativamente, em vez do fluxo de $\vec{B}$, podemos tomar a circulação de $\vec{A}$, e a força eletromotriz induzida num circuito é igual à variação temporal da circulação de $\vec{A}$. Mas, na verdade, $\vec{A}$ é um campo definido em todo espaço, e não pertencente necessariamente a pontos de um circuito. Vamos mostrar, como fizeram antes Ritz [6] e O'Rahilly [7], que, segundo a visão eletrônica da matéria, a indução deve ser atribuída à aceleração das cargas do circuito indutor. Tomemos o circuito metálico $C$, em que há uma corrente $i$ e achemos o potencial escalar e vetorial criado por todas as cargas do circuito no ponto $P$, Fig. 3 . A corrente será considerada como devida a uma densidade de carga linear $\lambda$ dotada de velocidade $v$. Sendo $\hat{t}$ o versor da tangente a $C$ num ponto do circuito, o vetor corrente será $\vec{i}=\lambda v \hat{t}$. Admite-se que o circuito é neutro, isto é, há cargas de compensação de sinal oposto, de forma que o campo eletrostático total se anula. Vejamos agora o potencial vetor das cargas móveis, Eq.12. No ponto $P$, o campo eletrostático criado por todas as cargas móveis do circuito é constante no tempo, porque uma carga ocupa, no instante seguinte, a posição ocupada pela carga a sua frente no instante anterior. Portanto, a componente de $\vec{A}$ devida às correntes de deslocamento se anula. A outra contribuição será

$$
\vec{A}(P)=\oint_{C} \frac{\lambda v \vec{t} d s}{r}=i \oint_{C} \frac{\hat{t} d s}{r}
$$

sendo $d s$ elemento de comprimento de arco de $C$. Se o circuito é fixo e a corrente constante no tempo, $\vec{A}(P)$ também será constante no tempo e o campo elétrico, obtido da Eq.12, será nulo. Notemos, de passagem, que o fato de o campo elétrico de indução ser zero para correntes estacionárias significa que a integral ao longo de $C$ dos termos de velocidade, na Eq.21, é nula. E isto explica por que $\vec{E}_{v}$, isto é, o campo elétrico dos EQ-P devido às velocidades, Eq.21, pôde ser sistematicamente ignorado no tratamento da indução entre circuitos $[6,7]$. Voltando à Eq.23, vemos que, se as cargas estão aceleradas, isto é, se $d i / d t \neq 0$, haverá no ponto $P$ o campo de indução $\vec{S}(P)$, que pela Eq.23, será dado por

$$
\vec{S}(P)=-\frac{1}{c} \frac{\partial \vec{A}}{\partial t}==\frac{1}{c} \frac{d i}{d t} \oint_{C} \frac{\hat{t} d s}{r}=-\lambda w \oint_{C} \frac{\hat{t} d s}{r}
$$

já que $\frac{d}{d t} \oint_{C} \frac{\hat{t} d s}{r}$ se anula.

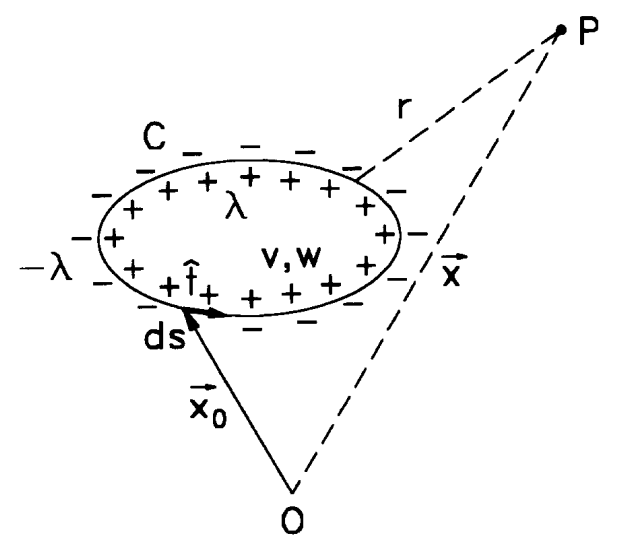

Figura 3. Cargas, com densidade linear $\lambda$ no circuito $C$, tem velocidade $v$ e aceleração $\mathrm{w}$ em presença de cargas de compensação. Elas criam no ponto $P$ o campo de indução dado pela Eq.23. 
Haverá também campo de indução em $P$ se $C$, embora com corrente constante no tempo, for móvel, por exemplo, se for dotado de velocidade de translação $\vec{u}$ (Fig.4). Neste caso, a velocidade das cargas móveis será $\vec{u}+v \hat{t}$ e das fixas, de sinal oposto, $\vec{u}$, de forma que para o potencial vetor, outra vez, só a velocidade $v \hat{t}$ dos portadores móveis em relação a $C$ dará contribuição. Outra vez, a parte devida às correntes de deslocamento se anula e o potencial vetor será dado pela Eq.23. Porém agora haverá campo elétrico de indução porque $\partial \vec{A}(P) / \partial t \neq 0$ pela translação do circuito (a menos que o ponto $P$ estivesse animado da mesma velocidade $\vec{u}$, anulando a variação do potencial vetor em $P)$.

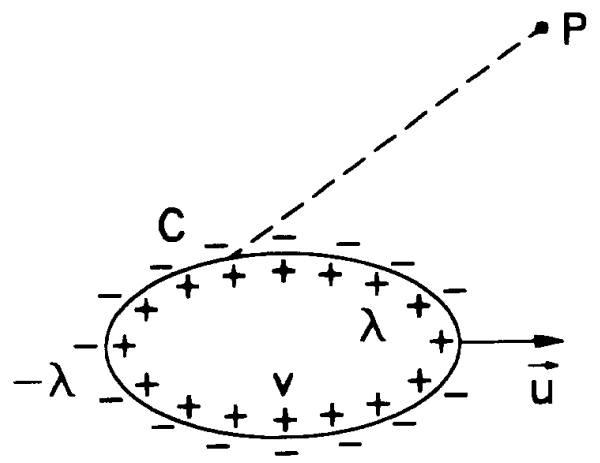

Figura 4. Esquema para o cálculo da indução em $P$ devido ao circuito $C$, com corrente, dotado de velocidade de translação $\vec{u}$.

Da mesma forma que o $\vec{E}_{v}$, o campo $\vec{E}_{w}$ devido à aceleração das cargas pôde ser ignorado ao longo dos anos pela preferência dada ao tratamento do fenômeno de indução como causado pela variação do fluxo 'aprisionado' no interior do circuito. Mas se o circuito fosse aberto? No cálculo presente, o potencial vetor é calculado no ponto $P$, sem necessidade de se pensar num circuito fechado que eventualmente o incluiria. Como dissemos, o cálculo de Ritz e O'Rahilly preenche um vazio que existe na didática usualmente empregada, que é a de começar o estudo da eletrostática e o da magnetostática com o campo de uma carga pontual, parada ou de um elemento de corrente, usando-se em seguida o princípio de superposição para abordar situações mais complexas. Mas este ponto de vista é esquecido no momento de se estudar a indução, como se esta fosse devida a um efeito coletivo do circuito indutor, ou seja, como se o princípio de superposição não pudesse ser aplicado. Por último, consideremos que $C$ está fixo e sua corrente é constante enquanto $P$ pertence a um circuito metálico em movimento. Neste aparecerá uma força eletromotriz devido agora à componente magnética, Eq.22, da força de Lorentz.

\section{Comentário e Agradecimen- tos}

Em [9], estudou-se com o auxílio da Eq.21 o caso da geração de diferença de potencial em uma barra metálica em repouso na presença de um ímã em movimento, abordado na literatura [2] através da variação de fluxo em inexistente circuito fechado. O autor já não corrobora o criticismo genérico e um pouco simplista expresso no artigo sobre o conceito de 'campo'.

$\mathrm{O}$ autor agradece ao $\mathrm{CNPq}$ a bolsa de produtividade, ao Prof. Renê Robert, da Universidade Federal do Paraná, pela troca de idéias sobre o assunto e ao Prof. Luiz Nunes de Oliveira pelos comentários a uma prévia versão deste.

\section{Apêndice}

Seja a carga $q$, com velocidade $\vec{v}$, que ocupa a posição $\vec{x}_{0}(t)$, no tempo $t$, ver Fig.5. Para o cálculo do potencial vetor no ponto $\vec{x}$, vamos dividir a Eq.11 em duas partes. Sejam $\overrightarrow{A_{1}}$ e $\overrightarrow{A_{2}}$ as parcelas

$$
\vec{A}_{1}(\vec{x})=\frac{1}{c} \frac{q \vec{v}}{\left|\vec{x}-\vec{x}_{0}(t)\right|}
$$

e

$$
\vec{A}_{2}(\vec{x})=\frac{1}{c} \int \frac{\frac{\partial \vec{h}\left(\vec{x}^{\prime}-\vec{x}_{0}(t)\right)}{\partial t}}{\left|\vec{x}-\vec{x}^{\prime}\right|} d V^{\prime}
$$

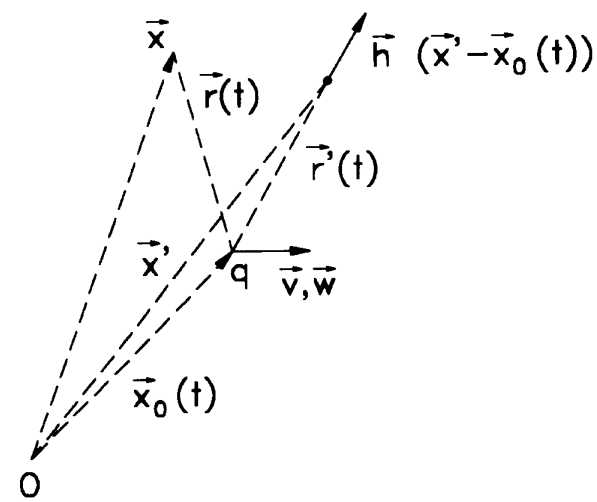

Figura 5. Esquema para o cálculo no ponto $\vec{x}$ do potencial vetor $\vec{A}_{2}$, Eq.A2, criado por uma carga $q$, em $\vec{x}_{0}(t)$ com velocidade $\vec{v}$ e aceleração $\vec{w}$, devido, às correntes de deslocamento $\partial \vec{h}\left(\vec{x}^{\prime}-\vec{x}_{0}(t)\right) / \partial t$ distribuidas no espaço.

a integral se estendendo a todo espaço, de elemento $d V^{\prime}$, sede da densidade de corrente de deslocamento $\partial \vec{h}\left(\vec{x}^{\prime}-\vec{x}_{0}(t)\right) / \partial t$. Para se calcular $\vec{A}_{2}(\vec{x})$, procedamos da seguinte maneira: 1) a derivada no tempo pode ser posta fora do integrando; 2) explicita-se $\vec{h}$ como o campo coulombiano de $q$ e faz-se

$$
\vec{h}(\vec{x})=-\nabla^{\prime} \frac{q}{\left|\vec{x}^{\prime}-\vec{x}_{0}(t)\right|}=\nabla_{0} \frac{q}{\left|\vec{x}^{\prime}-\vec{x}_{0}(t)\right|}
$$


e $\nabla^{\prime}$ e $\nabla_{0}$ significando gradientes tomados em relação às variáveis $\vec{x}^{\prime}$ e $\vec{x}_{0}$, respectivamente; 3 ) extrai-se agora $\nabla_{0}$ do integrando de $\overrightarrow{A_{2}}$. Consideremos, então, a integral

$$
\int \frac{q}{\left|\vec{x}^{\prime}-\vec{x}_{0}(t)\right|}
$$

que pode ser vista como o cálculo do potencial no ponto $\vec{x}$ criado pela distribuição esférica de cargas centrada em $\vec{x}_{0}(t)$, com densidade de carga inversamente proporcional à distância ao centro, $\left|\vec{x}^{\prime}-\vec{x}_{0}\right|$. Como a integral em Eq. A4 será uma função do módulo de $\vec{x}-\vec{x}_{0}$, digamos $F\left(\left|\vec{x}-\vec{x}_{0}(t)\right|\right)$, poderemos pôr

$$
\nabla_{0} \int \frac{q}{\mid \overrightarrow{\vec{x}^{\prime}-\vec{x}_{0}(t) \mid}} \frac{\vec{x}-\vec{x}^{\prime} \mid}{\mid \vec{x}} d V^{\prime}=-\nabla \int \frac{q}{\left|\vec{x}-\vec{x}_{0}(t)\right|} d V^{\prime}
$$

e o lado direito da Eq.5 é o campo elétrico da distribuição esférica mencionada no parágrafo anterior, no ponto $\vec{x}$. Para este, as cargas em pontos $\vec{x}^{\prime}-\vec{x}_{0}(t)$ exteriores à $\vec{x}-\vec{x}_{0}(t)$ não contribuem, enquanto que os interiores o fazem como se toda a carga estivesse concentrada em $\vec{x}_{0}(t)$. Chamando $\vec{x}^{\prime}-\vec{x}_{0}(t)$ de $\vec{r}^{\prime}(t)$ e $\vec{x}-\vec{x}_{0}(t)$ de $\vec{r}(t)$, vemos que a carga situada entre $\vec{x}_{0}(t)$ e $\vec{x}_{0}(t)+\vec{r}(t)$ é

$$
\int \frac{q}{\left|\vec{x}^{\prime}-\vec{x}_{0}(t)\right|} d V^{\prime}=q \int_{0}^{r} \frac{4 \pi r^{\prime 2} d r^{\prime}}{r^{\prime}}=2 \pi q r^{2}
$$

e o módulo do campo radial procurado será, então, $2 \pi q r^{2} / r^{2}=2 \pi q$ ou seja, um campo radial de módulo constante. Temos finalmente para $\overrightarrow{A_{2}}(\vec{x}, t)$, da Eq.A2

$$
\vec{A}_{2}(\vec{x})=\frac{1}{c} \int \frac{\frac{\partial \vec{h}\left(\vec{x}^{\prime}-\vec{x}_{0}(t)\right)}{\partial t}}{\left|\vec{x}-\vec{x}^{\prime}\right|} d V^{\prime}=\frac{q}{2 c} \frac{\partial}{\partial t} \hat{r}=\frac{q}{2 c} \frac{\partial}{\partial t} \frac{\vec{x}-\vec{x}_{0}(t)}{\left|\vec{x}-\vec{x}_{0}(t)\right|}
$$

Devemos agora retornar à Eq.12 e tendo em conta que

$$
\frac{\partial}{\partial t} \frac{1}{r}=\frac{\partial}{\partial t} \frac{1}{\left|\vec{x}-\vec{x}_{0}(t)\right|}=\frac{\vec{v} \cdot \vec{r}}{r^{3}}
$$

e

$$
\frac{\partial}{\partial t} \hat{r}=\frac{-\vec{v}}{r}+\frac{\vec{v} \cdot \hat{r} \hat{r}}{r}
$$

sendo $\vec{v}$ a velocidade de $q$. Usando-se agora as Eqs. A1, A2 e a Eq.11 e realizando-se as derivações, chegase então à Eq.21, sendo $\vec{w}$ a aceleração de $q$. Devemos notar que, como prevíramos, $\nabla \times \overrightarrow{A_{2}}=0$, por ser $\vec{A}_{2}$ um campo eletrostático radial só dependente de $r$, confirmando que correntes de deslocamento de campos eletrostáticos não criam campo magnético [8].

\section{References}

[1] J.B. Marion, Classical Electromagnetic Radiation, Academic Press, New York, 1967.
[2] J. Reitz, F. Milford e R. Christy, Fundamentos da Teoria Eletromagnética, tradução R. B. Sander e C. Duarte, Editora Campus, Rio de Janeiro, 3a. edição, 1982.

[3] R.P. Feynman, R. B. Leighton e M. Sands, The Feynman Lectures on Physics, Massachusetts, Addison-Wesley, 1965, vol. 2 .

[4] E. V. Bohn, Introduction to Electromagnetic Fields and Waves, Addison-Wesley Publ. Co., Reading, 1968.

[5] M. Jouget, Le champ électromagnétique, Librairie Armand Collin, Paris, 1949, 3a. parte.

[6] W. Ritz, Recherches Critiques sur l'Électrodynamique Générale, Gesammelte Werke, Gauthier Villars, Paris, 1911, pg.382.

[7] A. O'Rahilly, Electromagnetic Theory, Dover Publ. Inc., New York, Vol.1, Cap.7.

[8] R. C. T. da Costa e G. F. Leal Ferreira, Rev. Bras. de Física, 10, 353 (1980).

[9] G. F. Leal Ferreira, O. N. Oliveira Jr., Galilean Electrodynamics, 4, 54 (1993). 